\title{
EFFECT OF LOAD MATRIX METHOD IN REMOVING THE INTERFERENCE IN INTER AND INTRA CELLULAR WIRELESS NETWORKS
}

\author{
Banda Sreenivas ${ }^{1}$, S. Ramesh Babu ${ }^{2}$, S.Prabhakar ${ }^{3}$ And D.Karunakar Reddy ${ }^{4}$ \\ 1Department of Electronic \&Communication Engineering, Jyothishmathi Institute of \\ Technology \& Science, Thimmapur, Karimnagar, INDIA \\ targetsrinu8@gmail. com \\ 2 Department of Electronic \& communication Engineering, Nigama Engineering \\ College \\ Sambhayapally, Karimnagar,INDIA \\ ramesh_sag@rediffmail.com \\ 3 Department of Electronic \& communication Engineering, RRSCET, Hyderabad, \\ INDIA \\ saggurthi_plyahoo.co.in \\ 4 Department of Electronic \& communication Engineering, JNTU-Hyderabad, INDIA \\ karunakar.jntuhegmail. com
}

\begin{abstract}
Allocating resources to a mobile terminal over a short period of time, providing quality of service over wireless networks is the most stressing point for service providers. High degree of sharing is efficient, but requires service protection mechanisms to guarantee the Quality of service. In this paper we propose the multi cell interference on overall radio resource utilization. We also propose a joint management of interference within and between cells for allocation of radio resources, results are showing that there is a significant improvement in the resource utilization so that overall network performance is improved
\end{abstract}

\section{KEYWORDS}

Resource Allocation techniques, Load Matrix,

\section{INTRODUCTION}

Wireless communication is one of the most thrust areas in mobile cellular system which has evolved in many countries. The major challenge in cellular communication is supporting multimedia content and real-time services over wireless network. Wireless channel condition is affected by many factors such as fading, shadowing and interference which degrade the quality of the signal and cause delay, which will affect the total capacity of the system. In addition to this, two forms of interferences, inter-cell interference that occurs between cells and intra-cell interference caused by own users can be distinguished. In order to achieve efficient resource utilization in all sorts of deployment scenarios and Q o S requirements in the future wireless cellular systems, the new resource allocation method must be developed. 
The main difference between uplink and downlink transmissions is the fact that in the first case each user is affected by its individual channel, whereas the signals arriving at a certain mobile are passed through the same channel in the downlink, but need a perfect power control that ensures the same power level for all users at the receiver. If multiple access or co-channel interference in cellular networks disturbs the transmission, interferers that are separable in space can be suppressed with multiple antennas, resulting in an improved Signal to Interference plus Noise Ratio (SINR). Assessing the quality of resource allocation, one can distinguish cell capacity achieved in the system with fairness amongst users, in terms of offered transmission opportunities. The trade-off between cell capacity and fairness is very important in a scheduling algorithm while allocating resources. The objective of the allocation scheme is to maximize total network utility can be utilized for optimal resource allocation. [1] In this paper, we discuss inter cell interference problem of scheduling process by introducing load matrix concept and using HSUPA.[4][6].

\section{INTERFERENCE IN CELLS}

A cellular system consists of many cells with channels (timeslots, bandwidth, or codes) reused at spatially separate locations. Due to the fundamental nature of wireless propagation, transmissions in a cell are not limited to within that cell, and thus there is inter cell interference between users and base stations, that use the same channels. The majority of current systems are interference limited rather than noise limited. Interference is part of every mobile cellular communications system, and it constitutes a limitation to both radio network capacity and quality of service provided to users [3]. Inter cell interference is managed via averaging of the effects of multiple interferers. It is more effective in the uplink than in the downlink. Interference averaging also allows statistical multiplexing of busty users, thus increasing system capacity.

Resource allocation schemes in the uplink are of two categories, distributed and centralized. The objective of distributed allocation is to reduce the complexity to the Radio Network Controller (RNC). This scheme does not know the channel conditions of adjacent cells. Where as in case of centralized schemes, the network controller is responsible for allocating the resources in every cell.On the forward link, the data is split by the RNC to a number of base stations and the received data is combined by the mobile terminal. On the reverse link, the participating base stations forward the received data to the serving RNC to combine. [12]

In interference limited systems, the uplink capacity is limited by the total transmitted power at the base station and this power was limited by uplink capacity. Inter cell interference calculation is done by multiplying the number of users in a cell by the average interference offered in this cell, this kind of calculation, being suitable for real-time interference simulations based on the number of users, their path loss, slow fading, and the cell area. But in uplink, inter cell interference density analysis is performed by assuming perfect power control. The number of users is taken into account, as well as the received signal power and the activity factor according to the user's service calculates the average inter cell interference per cell. [7]

Usually, in radio network planning, a fixed value is taken for the inter-to-intra cell interferences ratio ( $i$ factor) 0.65 , as well as for the interference margin in the power budget is $3 \mathrm{~dB}$. The inter cell interference will be particularly significant when inter cell users are near the cell boundary and when the frequency reuse factor is equal to one, hence it is a crucial factor and cannot be ignored in the scheduling process for future wireless cellular systems. 


\subsection{Interference Model}

The performance of a high SINR user is dominated by intracell (or inter-user) interference, increasing number of users also results in increased intracell interference and an orthogonal access benefits by eliminating the intracell interference. We know that as the other-cell to owncell signal ratio increases, the performance is dominated by other-cell interference rather than only by intracell interference. [8] Therefore, in case of heavy inter-cell interference, the gains of orthogonal access over non-orthogonal access go down.

\subsection{Intra - Cell Model}

The intracell interference limits the maximum achievable data rates and limits the capacity of the uplink. This model calculates the average inter-cell interference per cell, being necessary to use a user distribution in the cell area. The calculation of the intra-cell interference in Down link, on $\mathrm{MT}_{i}$ is given by equation 1

$$
\mathrm{I}_{\text {Intra,j }}^{\mathrm{DL}}=\left(\mathrm{P}_{\mathrm{Total}, \mathrm{BS}}-\mathrm{P}_{\mathrm{BS} \rightarrow \mathrm{MT} \mathrm{T}_{\mathrm{i}}}\right) \times \propto \times \mathrm{L}_{\mathrm{BS} \rightarrow \mathrm{MT}_{\mathrm{i}}}[\mathrm{W}]
$$

Where $P_{\text {Total } B S}$ is the total power transmitted by the Base Station (BS), $P_{B S \rightarrow M T i}$ is the power transmitted by the BS to the (Mobile Terminal) MT in which interference is being calculated, and $L_{B S \rightarrow M T i}$ is the propagation loss between BS and MT. The orthogonality factor $\alpha$ can take values between $0-1$. In Uplink Interference is given by equation 2

$\mathrm{I}_{\mathrm{Intra,j}}^{\mathrm{UL}}=\sum_{\mathrm{g}=1}^{\mathrm{G}} \mathrm{P}_{\mathrm{BSj} \leftarrow \mathrm{MT}} \times \eta \mathrm{g} \times \mathrm{N}_{\mathrm{j}, \mathrm{g}} \quad[\mathrm{W}]$

Where $P_{B S j \leftarrow M T}$ is the power received at BS ${ }_{j}$ from an MT, $\eta_{g}$ is the activity factor of service $g, N$ $j, g$ is the number of MTs using service $g$ on the cell of $\mathrm{BS}_{j}$, and $G$ is the total number of services used.

\subsection{Inter-Cell Model}

Power control on the down link has less impact on inter cell interference than on the uplink because the downlink transmissions all originate from the cell center. Whereas Uplink transmissions can come from the cell boundaries. Hence need to focus more on the effect of power control on the uplink. In DL, the model used for inter-cell interference, in an MT using a service $\mathrm{g}$ is given by,

$I_{\text {Inter,j }}^{\text {DL }}=\sum_{j=2}^{N_{B S}} P_{\text {Total,BSj }} \times \propto r_{j}^{-a} \times 10^{\Delta L j / 10}[\mathrm{~W}]$

where $\mathrm{P}_{\text {total }}, \mathrm{BS}_{\mathrm{j}}$ is, BS j's total transmitted power, $\mathrm{N}_{\mathrm{BS}}$ is the number of interfering BS's, $r_{j}{ }^{-a}$ is the path loss, $a$ representing path loss exponent, $\Delta L_{j}$ is associated to slow fading, following a statistical distribution with zero mean and a certain standard deviation, and $r_{j}$ represents the distance between the interfering $\mathrm{BS}_{j}$ and the MT.

$\mathrm{I}_{\mathrm{Inter}, \mathrm{j}}^{\mathrm{UL}}=\sum_{K=1, K=j}^{N_{B S}} \sum_{g=1}^{G} P_{B S j} T_{M K} \times \eta \mathrm{g} \times \mathrm{N}_{\mathrm{k}, \mathrm{g}} \times \mathrm{A}[\mathrm{W}]$

$\mathrm{P}_{\mathrm{BSj}} \leftarrow \mathrm{T}_{\mathrm{Mk}}$ is $\mathrm{BS}_{j}$ power received from the MT that is being covered by an adjacent cell $k, N_{k, g}$ is the number of users using service $g$ in interfering cell $k$. A larger interference reduces SINR and hence increases user BER. Intercell interference can be reduced by separating cells operating on the same channel by a large distance. 


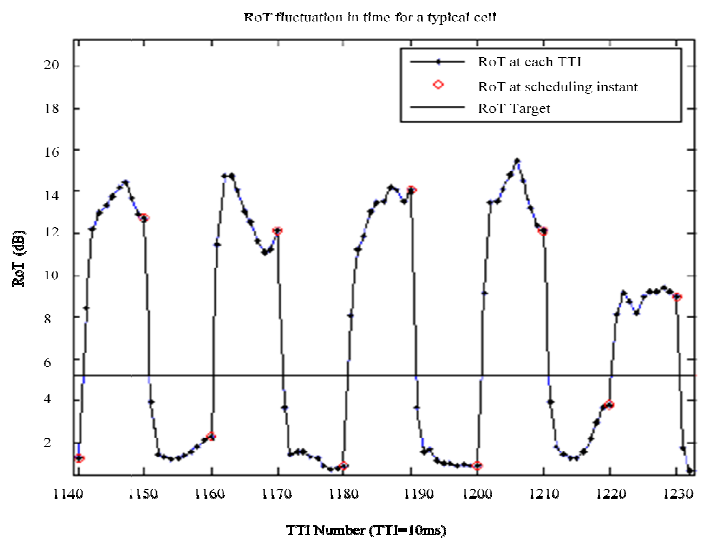

Figure 1. RoT fluctuation in multicell.

Good cellular system designs are interference limited that is the interference power is much larger than the noise power. Figure. 1 shows the RoT fluctuation due to inter cell interference in a typical cell. The RoT of a cell dramatically increases to above the threshold and rapidly decreases to below the threshold while allocating resources to users. But for ideal performance in terms of interference management this RoT should be close to threshold $\left(\right.$ RoT $\left._{\text {target }}\right)$. As the uplink load increases user terminals have to their transmit power substantially to overcome the increased interference level at the base station. Due to the fact that interference cell, the transmit power of user terminals is limited by total received power at the base station actually limits the uplink capacity. [5]

\section{RESOURCE ALLOCATION}

In next generation networks a variety of services with different requirements are in demand, like real time communications, broadband Internet access, email services are expected. Consequently, packet scheduling mechanisms and resource allocation techniques for QoS guarantees will play an important role. The Radio resource allocation is a challenging problem in wireless networks due to different channel conditions of user and the main aim of resource allocation is to assign radio resources to individual users in order to achieve maximum capacity while meeting the required quality of service. A contiguous resource allocation scheme is defined for both the uplink and the downlink.

In uplink the distributed and centralised allocation schemes reduce the complexity of network. The resource allocation problem in these systems causes inefficient use of radio spectrums and to utilize multiple and maximize the system capacity, but they have to consider admission and access control in conjunction with resource allocation mechanism, subcarriers in wireless systems such as OFDM (orthogonal frequency division multiplexing)[9][14]. Allocating different number of subcarriers intelligently, the inefficiency issue can be handled. In order to provide various choices of scheduling performance and signalling overhead, multiple resources allocation types are defined.

In multiuser OFDMA systems, multiuser diversity can be easily achieved by the allocation of sub channels to users, and these channels are independent for each user, with this the resource allocation problem for multiuser OFDMA systems has been extensively investigated. The quality of resource allocation can be assessed by overall throughput and fairness. In a wireless network environment the trade-off between throughput and fairness is important for scheduling. Due to 
International Journal Of Advanced Smart Sensor Network Systems ( IJASSN ), Vol 2, No.3, July 2012

the nature of resource allocation (time and frequency), transmissions suffer no interference from within the cell and further see minimal interference from neighbouring cells.[11]

\section{CONCEPT OF LOAD MATRIX}

The Load Matrix (LM) concept has the facility to joint management of interference within and between cells while allocating radio resources to users and this concept proposed intakes the intercell interference information into account in order to avoid RoT outage. In a multicell system one of the main challenge in resource allocation is the control of intercell interference. LM is a centralized scheduler, uses a database containing the load contribution of all active users in the network and it assigns radio resources to all active users in the network. The basic problem in the uplink scheduler is to assign appropriate transmission rate and time to all active users, result maximum radio resource utilization across the network while satisfying the QoS requirements of all the users. [13]

The important factor in the resource allocation is the users transmit power. The constraints to be satisfied for a network of $\mathrm{M}$ users and $\mathrm{N}$ cells are

Constraint1: This constraint states that the maximum user power $P_{i, \max }$. For each active user $i$ in the network, its transmit power $\mathrm{P}_{\mathrm{i}}$ must be maintained in an acceptable region defined as

$$
0 \leq \mathrm{P}_{\mathrm{i}} \leq \mathrm{P}_{\max } \quad \mathrm{i} \in\{1, \ldots \ldots, \mathrm{M}\}
$$

Constrain2: The total received power at base station should be kept below a certain threshold for all $\mathrm{N}$ base stations in the network it uses Rise over Thermal noise (RoT) to represent the interference constraints.

$\operatorname{RoTj} \leq \operatorname{RoT}_{\text {target }} \mathrm{j} \in\{1, \ldots \ldots \ldots, \mathrm{N}\}$

RoTj is the total in band received power fixed target value to maintain uplink interference level at the base station $\mathrm{j}\left(\mathrm{BS}_{\mathrm{j}}\right)$ over thermal noise. The RoTj for $\mathrm{M}$ active users in the network given below is used to estimate RoT of cells, can be written as

$$
\mathrm{ROT}_{\mathrm{j}}=\left(N^{\prime}+\sum_{i=0}^{M} \mathrm{P}_{\mathrm{i}} \mathrm{G}_{\mathrm{ij}}\right) / N^{\prime}
$$

Constraint 3 : The signal to noise plus interference ratio required at the serving base station $\mathrm{j}$ if rate $\mathrm{k}$ is being assign to the user to achieve a given frame error rate is $\mathrm{SINR}_{\mathrm{target}, \mathrm{k}}$. For each user, depending on its channel type and speed, each rate $\mathrm{k}$ has a minimum required SINR called $\mathrm{SINR}_{\text {target,k}}$. This constraint satisfies only by considering SINR $_{\text {target, } \mathrm{k}}$ as SINR.

$\operatorname{SINR}_{\mathrm{i}, \mathrm{Z}} \geq \operatorname{SINR}_{\text {target, } \mathrm{k}} \mathrm{i} \in\{1, ., \mathrm{M}\}, \mathrm{k} €\{1, \ldots, \mathrm{K}\}$ 


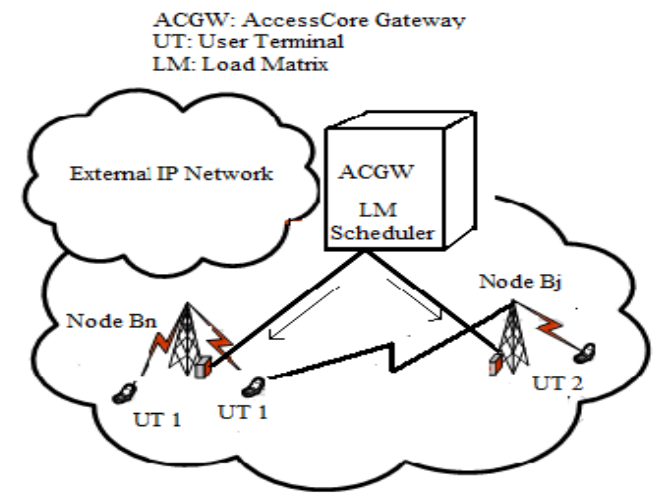

Figure 2. Centralize LM Scheduling in a 3G LTE System.

LM scheduling can be implemented in both centralized and decentralized strategies. In a central scheduler entity assigns radio resources to all the users in the network where as in decentralized scheduling each base station implemented with identical LM scheduling, Figure. 2 is implementation of LM scheduling for the $3^{\text {rd }}$ Generation Long-Term Evolution (3G LTE).

A centralized scheduler assigns radio resources to all the $\mathrm{M}$ users and $\mathrm{N}$ cells in the network, LM $\mathrm{i}, \mathrm{j}$ is the load factor contribution by user $\mathrm{i}$ at BS $\mathrm{j}$ defined as

$$
\mathrm{LM}_{\mathrm{i}, \mathrm{j}}=\frac{\mathrm{P}_{\mathrm{i}} \mathrm{G}_{\mathrm{ij}}}{N^{\prime}+\sum_{m=1}^{M} P_{m} G_{m j}}
$$

Where $G_{i j}$ is the channel gain from user i to $B S_{j}$ averaged over scheduling period, $N^{\prime}$ is the thermal noise and $P_{i}$ is the transmitted power. The $L_{M}$ i,j values stored in column $\mathrm{j}$ of $\mathrm{LM}$

database, RoT of cell $\mathrm{j}$ can be written $\mathrm{ROT}_{\mathrm{j}}=\frac{1}{1-\sum_{i=1}^{M} \mathrm{LM}_{\mathrm{ij}}}$

$\operatorname{SINR}_{\mathrm{i}, \mathrm{j}}$ can be written as

$\operatorname{SINR}_{\mathrm{i}, \mathrm{j}}=\frac{\mathrm{P}_{\mathrm{i}} \mathrm{G}_{\mathrm{ij}}}{N^{\prime} R o T_{j}-\mathrm{P}_{\mathrm{i}} \mathrm{G}_{\mathrm{ij}}}$

The required transmitted power for user $\mathrm{i}$ at rate $\mathrm{k}$ is,

$\mathrm{P}_{\mathrm{i}, \mathrm{k}}=\frac{N^{\prime} R o T_{\text {target }}}{\mathrm{G}_{\mathrm{ij}}} \frac{\text { SINR target, } \mathrm{k}}{1+\mathrm{SINR} \text { target, } \mathrm{k}}$

If above all constraints are satisfied then only power $\mathrm{P}_{\mathrm{i}, \mathrm{k}}$ is acceptable and user $\mathrm{i}$ will be scheduled for transmision. After that LM elements are updated and RoT is calculated for each cell using [10]. The performance of the LM scheduling has the best RoT over other algorithms because this scheduler significantly reduce the probability of the RoT exceeding its target. Priority functions are used to rank the uers in the scheduling process and make a balance between cell throughput and fairness.

A priority function in LM scheduling is introduced based on users load vector that includes intra and inter cell impact on the network and it tries to maximise the network capacity through these interference managements. In each base station the LM allocation process simultaneously 
increases allocated resources to avoid interference imbalance among the cells. A user with higher channel gain has highest priority it is evident that giving priority to a user with better channel condition increases the cell throughput but in a multicell network could have severe impact on the throughput of other cells. Hence a new priority approach to load matrix is the Global Proportional Priority (GPP) considers interference contribution of the user to other cells is defined as

Priority $_{\mathrm{i}}=\frac{\mathrm{G}_{\mathrm{ij}}}{\sum_{n=1, n \neq j}^{M} \mathrm{G}_{\mathrm{i}, \mathrm{n}}} \forall i \in\{1, \ldots M\}$

Where $\mathrm{G}_{\mathrm{i}, \mathrm{j}}$ is the total channel gain from under i to $\mathrm{BS}_{\mathrm{j}}$ averaged over the scheduling period. LM process uses Capacity Check (CC) process to assign rates to the highest priority user in each cell to update LM elements and while performing capacity checking it maintains overall performnace of the network. The CC operates on small margin around RoT target instead of a fixed RoT target threshold result much improved interference outage performance and higher resource utilization.[15]

\section{SIMULATION RESULTS}

To evaluate the performance of the LM concept a HSUPA system simulator is used in a simulation scenario consisting of 16 cells with 8 users in each cell randomly and uniformly distributed. The RoT is set at $5.23 \mathrm{~dB}$ with carrier frequency of $2 \mathrm{GHz}$ and simulation time $20 \mathrm{sec}$. LM scheduler has the capability to maintain RoT not to exceed its target. LM scheduling is compared with Benchmark algorithm [2] to show effictiveness of Load Matrix concept and to carry the performance of resource allocation in terms of interference outage probability and average cell throughput. The user data, BPSK modulated signal and RoT response have been shown in figures 3,4 and 5. The figures 6,7 and 8 are for Cumulative Density Function (CDF) of RoT of inter cell, CDF of intra cell and Probability density function (PDF) of RoT. These diagrams demonstrate considerable performance in interference outage compared to benchmark algorithm in terms of cell th

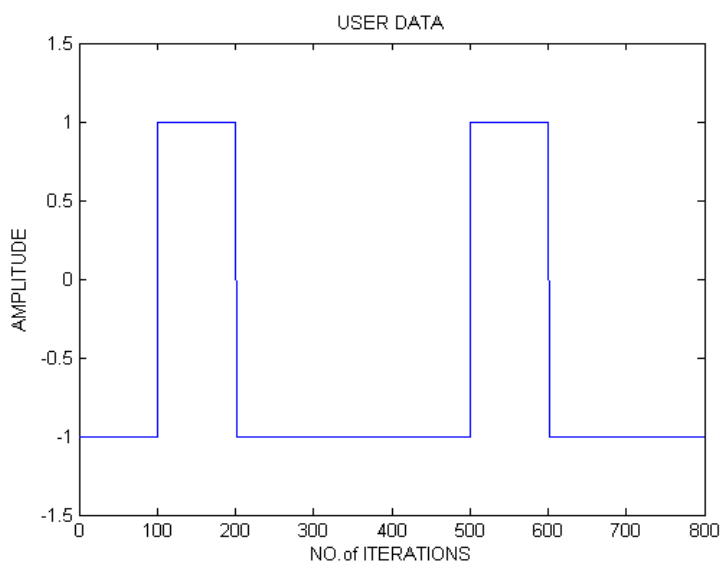

Figure. 3 User data 
International Journal Of Advanced Smart Sensor Network Systems ( IJASSN ), Vol 2, No.3, July 2012

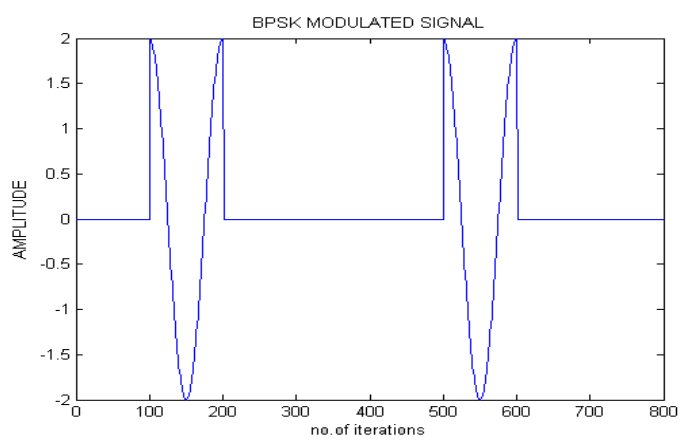

Figure 4. BPSK Modulated Signal

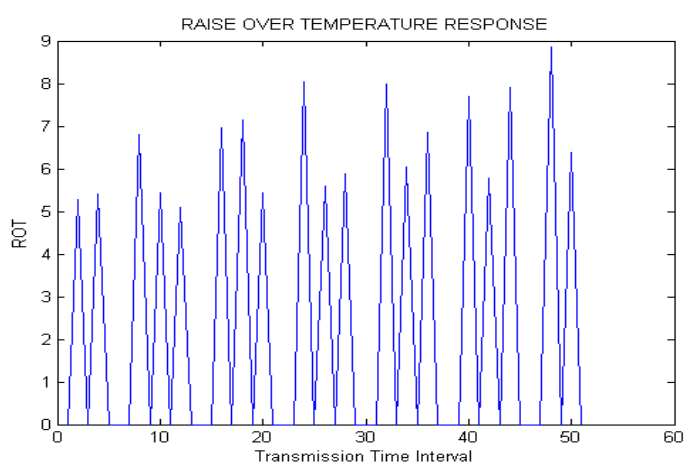

Figure 5. ROT Response

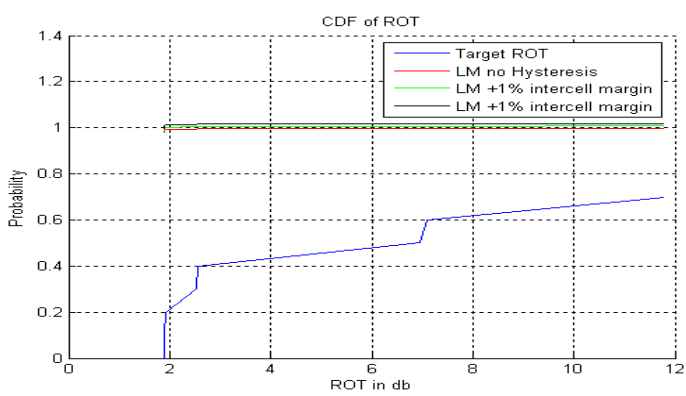

Figure 6. CDF of RoT for Inter cell Interferen

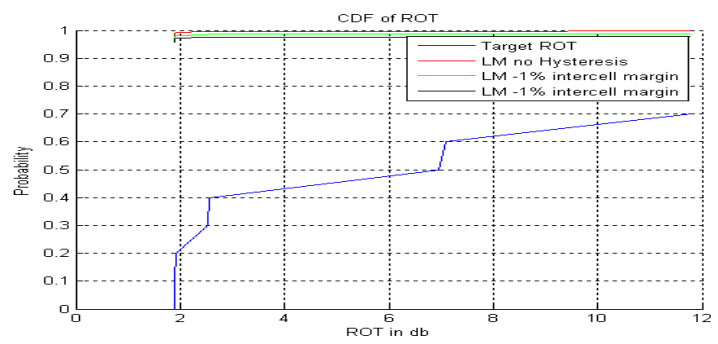

Figure 7. CDF of RoT for Intra cell Interference 
International Journal Of Advanced Smart Sensor Network Systems ( IJASSN ), Vol 2, No.3, July 2012

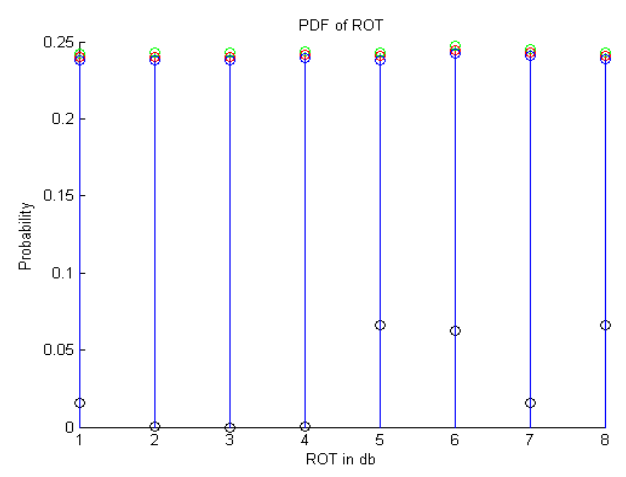

Figure 8. PDF of ROT

\section{CONCLUSION}

In this work we have evaluated system capacity and fairness performance of several transmission schemes with LM scheduling. LM concept is presented specifically to provide an efficient resource allocation by jointly considering inter cell and intracellular interferences before allocating radio resources. A novel approach towards efficient uplink scheduling is presented. We have developed a system level simulator for HSUPA system based on the proposed simulation conditions. The effect on the scheduling performance can be observed in the simulation results provided and these results indicate that selection of RoT as well as transmit power significantly affect the performance. By incorporating a new concept of separate margins for inter cell and intra cell interferences in the LM has better control over interferences results high overall network performance.

\section{REFERENCES}

[1] L. Wu and Z. Tian, "Capacity-Maximizing Resource Allocation for Data-Aided Timing and Channel Estimation"IEEE International Conference on Acoustics, Speech and Signal Proceeding, Montreal, Canada, vol. 4, pp. 525-528, May 2004.

[2] 3GPP TR 25.896, "Feasibility Study of Enhanced Uplink for UTRA FDD V6.0.0," www.3gpp.org, Mar. 2004.

[3] 3GPP, TS 36.211, "Evolved Universal Terrestrial Radio Access (E-UTRA); Physical Channels and Modulation (Release 8)".

[4] H. Holma and A. Toskala, WCDMA for UMTS: Radio Access for Third Generation Mobile Communications, second edition. John Wiley \& Sons, 2002.

[5] Hassibi B. and Hochwald B. M., "High-rate codes that are linear in space and time," IEEE Transactions on b Information Theory, vol. 48, no. 7, pp. 1804-1824, 2002.

[6] Khan, F., "A time-orthogonal CDMA high speed uplink data transmission scheme for 3G and beyond," IEEE Communication Magazine, pp. 88-94, Feb. 2005

[7] D. Tse and P. Viswanath. Fundamentals of wireless communication. Cambridge University Press, Cambridge, UK, 2005.

[8] Andrea Goldsmith, wireless communications, Cambridge, 2005.

[9] Farooq Khan, "LTE for 4G mobile broadband", Cambridge: Cambridge University Press, 2009.

[10] S. Moshavi, "Multi-user detection for DS-CDMA communications," IEEE Communication Magazine, vol. 34, pp. 124-136, Oct. 1993.

[11] M. K. Varanasi and B. Aazhang, "Multistage Detection in Asynchronous Code Division Multiple access communications," $\quad$ IEEE Trans. Commun., vol. 38, pp. 509-519, Apr. 1990.

[12] Joon Bae Kim and Micheal L.Honig "Resource allocation for Multiple classes of DS-CDMA Traffic,’IEEE Transactions On Vehicular Technology, vol.49, no.2, pp. 506-518, March 2000. 
International Journal Of Advanced Smart Sensor Network Systems ( IJASSN ), Vol 2, No.3, July 2012

[13] Saied Abedi, "Efficient Radio Resource Management for Wireless Multimedia Communications," IEEE Transactions Wireless Communications, vol.4, no.6, pp. 2811-2822, March 2005.

[14] U. Saraydar, Narayan B.Mandayam, "Pricing and Power Control in a Multicell Wireless Data Network,’IEEE Journal on Communications,” vol.19, no.10, pp. 1883-1892,

[15] Hannes Ekstrom, Anders Furuskar, Stefan Parkvall, "Technical Solutions for the 3G Long-Term Evolution,’IEEE Communications Magazine, pp 38-45, March 2006.

\section{Authors short Biography}

BANDA SREENIVAS is working as an Associate professor in Department of Electronics and Communication Engineering in JYOTHISHMATHI INSTITUTE OF TECHNOLOGY AND SCIENCE, Karimnagar, and Affiliated to JNTU-Hyderabad, INDIA. Obtained B.Tech from KITS-Warangal, Received M.Tech in Digital Systems and Computer Electronics from JNTU-Hyderabad. Published and presented THREE papers in the International conferences and TWO papers in National conferences. Has 15 years of experience in teaching and industry together. He is the Reviewer for three international journals, life member of ISTE, Member IAENG and Member IACSIT

SAGGURTHY RAMESH BABU working as a professor and HOD ,in Department of Electronics and Communication Engineering in NIGAMA ENGINEERING COLLEGE Karimnagar.,B.Tech from pullareddy engg.college karnool, completed M.Tech from JNTUKakinada and published many papers in an International conferences/National conferences. have around 15 years of experience in teaching life member of ISTE

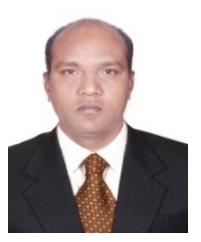

SAGGURTHY PRABHAKAR RAO received his Bachelor of Engineering (B.E.in E.C.E) in electronics and communications engineering from Vasavi College of Engineering Hyderabad in 1991 and Master of Engineering in Communications from C.B.I.T Hyderabad in 2005. Up to 2008 he was the Associate Professor in ECE department of Sri Devi Women's Engineering College, Hyderabad. joined in RRSCET as Professor in 2008 after that he taken many responsibilities as Head of the Department, VicePrincipal, In-Charge for inspections of AICTE, SBTET, JNTUHAs Prof. \& HOD he conducted and attended many seminars, workshops, conferences. He was enrolled his $\mathrm{PhD}$ Course and doing his research work with JNTUH in 2007 onwards in wireless communications and completed his Pre-PhD course and published 3 internationals journals and 3 national conferences and participated in one international conference. Research interests include communications, wireless networks, security and quality of service in wireless communications. He is currently working for wireless security and quality of service in wireless communications.

D.KARUNAKAR REDDY is working as a lecturer in JNTU Hyderabad Research scholar in Department of Electronics and Communication Engineering in JNTU Hyderabad completed graduation in 2002 completed his M.Tech in 2005.having 5 years experience in IT industry
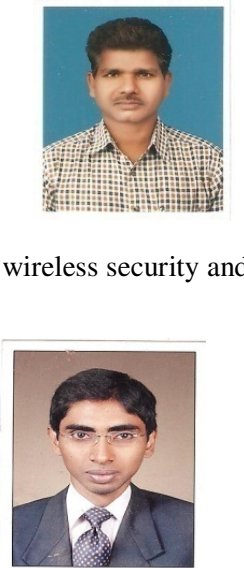